Изв. Крымск. Астрофиз. Обсерв. 116, № 2, 49-61 (2020) doi:10.31059/izcrao-vol116-iss2-pp49-61

УДК 523.98

\title{
Распределенный электрический ток и его связь с ультрафиолетовым излучением активной области
}

\author{
Ю.А. Фурсяк, А.С. Куценко \\ ФГБУН “Крымская астрофизическая обсерватория РАН”, Научный, Крым, 298409 \\ yuriy_fursyak@mail.ru
}

Поступила в редакцию 7 июля 2020 г.

\begin{abstract}
Аннотация. Используя магнитограммы компонент вектора магнитного поля на уровне фотосферы, получаемые инструментом Helioseismic and Magnetic Imager (HMI), установленным на борту космического аппарата Solar Dynamics Observatory (SDO), вычислены вертикальные электрические токи для активной области NOAA 12192 за период с 22 по 25 октября 2014 года с временным разрешением 12 минут. Выявлено наличие в исследуемой активной области (AО) крупномасштабной токовой структуры - распределенного электрического тока, имеющего абсолютные значения в диапазоне (40-90)·10 12 А. Предполагается, что распределенный ток охватывает всю АО, и, выходя в верхние слои солнечной атмосферы в одной части области, замыкается через хромосферу и корону на оставшейся ее части. Для проверки этого предположения проанализирована связь временных изменений величины распределенного тока с уровнем корональной активности, а также с интенсивностью ультрафиолетового излучения (УФ) АО в диапазонах длин волн $1600 \AA, 304 \AA, 193 \AA$ и $94 \AA$. Показано, что: 1) Временные интервалы максимумов величины распределенного тока совпадают по времени с повышенной вспышечной активностью АО. Отсутствие резких изменений величины распределенного тока во время вспышек может быть объяснено высокой индуктивностью токонесущих петель. 2) Грубая оценка магнитной энергии, выносимой распределенным током в корону, дает для AO NOAA 12192 значения $10^{33}-10^{34}$ эрг. Только небольшой объем этой энергии реализуется во время вспышечных процессов в АО, большая ее часть тратится на иные диссипативные процессы в короне. 3) Сравнение временных вариаций интенсивности излучения в канале $193 \AA$ с динамикой распределенного тока в АО показывает хорошую взаимосвязь этих величин (коэффициент корреляции $k=0.63)$. В то же время отсутствует корреляция между величиной распределенного тока и интенсивностью УФ-излучения в диапазонах $1600 \AA$, $304 \AA$ и $94 \AA$. 4) Полученные нами результаты, возможно, могут объясняться концепцией LRC-контура токонесущей корональной петли, предложенной в 1967 году Альфвеном и Карлквистом и развитой в работах В.В. Зайцева, А.В. Степанова и др. Согласно данной модели, крупномасштабные электрические токи должны существовать в верхних слоях солнечной атмосферы и принимать участие в нагреве коронального вещества.
\end{abstract}

Ключевые слова: Солнце, активные области, солнечная активность, электрические токи, нагрев короны

\section{1 Введение}

Несмотря на большие массивы поступающей информации (ежедневные данные одного только инструмента Atmospheric Imaging Assembly (AIA, Lemen et al., 2012), установленного на борту космического аппарата Solar Dynamics Observatory (SDO), составляют около 2 Тбайт), наличие современных наземных и космических (например, SOHO, Hinode, TRACE, SDO и др.) телескопов, проблема нагрева солнечной короны остается одной из нерешенных задач современной физики Солнца.

Разные авторы выделяют целый ряд механизмов нагрева коронального вещества, которые можно разделить на две большие группы - внешние (свободная магнитная энергия привносится извне) и внутренние (нагрев плазмы осуществляется за счет конвертации свободной энергии магнитной петли). K внешним механизмам нагрева относятся испарение хромосферной плазмы в 
корону (Antonucci et al., 1998; Bornmann, 1998), диссипация альфвеновских волн (Hollweg, 1984; Ionson, 1984), циклотронное поглощение генерируемых во время вспышки электромагнитных волн (Melrose, Dulk, 1984) и др. Среди внутренних механизмов нагрева коронального вещества можно выделить микровспышки, обусловленные пересоединениями внутри магнитной петли (Parker, 1988), тиринг-неустойчивость (Galeev et al., 1981) и омическую диссипацию электрических токов (Spicer, 1991).

Накопление электрических токов в короне может быть реализовано двумя путями. Первый из них предполагает формирование электрических токов на уровне фотосферы в результате скручивания или сдвига магнитной трубки (McClymont, Fisher, 1989; Török, Kliem, 2003; Aulanier et al., 2005; Dalmasse et al., 2015). Второй механизм подразумевает скручивание магнитной трубки еще в глубине конвективной зоны, на этапе ее формирования или подъема (Cheung, Isobe, 2014), что приводит к всплытию магнитного потока с уже сформированной собственной системой электрических токов (Leka et al., 1996; Longcope, Welsch, 2000).

Существование электрических токов в атмосфере Солнца на протяжении значительных временных интервалов предполагает замкнутость электрической цепи с током. Ряд теоретических моделей (Parker, 1996; Aulanier et al., 2005; Török et al., 2014; Dalmasse et al., 2015) предсказывают замыкание такой цепи на уровне фотосферы и наличие в магнитной трубке как прямых, так и обратных электрических токов (электрические токи в такой магнитной трубке должны быть скомпенсированными). Однако данные наблюдений указывают на то, что электрические токи в отдельных полярностях магнитного поля активной области (AO) в большинстве случаев не являются в полной мере скомпенсированными (Georgoulis et al., 2012). Особенно это касается областей с высокой вспышечной продуктивностью (Liu et al., 2017; Kontogiannis et al., 2017). Более того, обнаружение обратных токов в АО является большой проблемой, и на сегодня можно найти лишь немногие работы, в которых есть указания на их существование (см., например, Gosain et al., 2014). Melrose (1991) объясняет сложность обнаружения обратных токов либо очень низкими абсолютными величинами (ниже порога обнаружения) этих токов, которые к тому же распределены на значительной площади в АО, либо тем, что эти токи сконцентрированы на небольших, неразрешенных площадях. Альтернативное объяснение, которое рассмотрим более детально, состоит в том, что обратные токи могут располагаться ниже уровня фотосферы (см. рис. 3 в Melrose, 1995).

Впервые идея представить токонесущую петлю как аналог замкнутого электрического контура с замыканием токов под фотосферой была сформулирована в 1967 году (Alfven, Carlqvist, 1967). Концепция базировалась на первых измерениях вертикальных электрических токов в фотосфере, выполненных А.Б. Северным (Severny, 1964). В дальнейшем предположение о том, что электрические токи могут замыкаться ниже уровня фотосферы, можно увидеть в работе (Абраменко и др., 1991), а сама модель эквивалентного электрического (LRC) контура для описания процессов в токонесущей корональной петле была развита в работах В.В. Зайцева, А.В. Степанова и других авторов (Zaitsev, Khodachenko, 1997; Zaitsev et al., 1998; Zaitsev, Stepanov, 2008; Stepanov et al., 2012; Zaitsev, Kronshtadtov, 2016). Согласно этой модели, электрические токи генерируются конвективными движениями плазмы на уровне фотосферы, или, в случае расположения основания магнитных петель в области пятен, вращательными движениями самих пятен со скоростью порядка 0.1 км с (Stenflo, 1969). Сгенерированный на уровне фотосферы электрический ток далее течет через хромосферу и корону от одного основания петли к другому. Поскольку, как было показано выше, явных проявлений обратных токов не обнаружено, предполагается, что обратный ток течет ниже уровня фотосферы по кратчайшему пути от одного основания петли к другому, замыкая тем самым контур.

Расчеты, выполненные в рамках контурной модели токонесущей магнитной трубки показывают, что конвективные движения в фотосфере со скоростью порядка $0.1 \mathrm{kм} \mathrm{c}^{-1}$ приводят к генерации тонких магнитных трубок радиусом 100-1000 км и электрическим током внутри величиной порядка $10^{11}-10^{12}$ A (Zaitsev, Khodachenko, 1997; Khodachenko, Zaitsev, 2002). Эти расчеты хорошо согласуются с результатами, представленными в Zaitsev et al. (1998), где на основе данных наблюдений на Metsahovi Radio Research Station в миллиметровом диапазоне, для 16 вспышечных событий получены значения электрических токов $10^{11}-1.42 \cdot 10^{12} \mathrm{~A}$, а также рядом других наблюдательных данных.

Так как сгенерированные в глубине конвективной зоны или же на уровне фотосферы крупномасштабные электрические токи уходят высоко в корону, то должны наблюдаться различные процессы и явления, связанные с диссипацией этих токов в верхних слоях солнечной атмосферы. Стереоскопический анализ корональных магнитных петель, проведенный в конце 20-го века с помощью 
инструментов космического аппарата $\mathrm{SOHO}$, позволил обнаружить лишь незначительные изменения температуры корональных петель с высотой (Aschwanden et al., 1999, 2000). Аналогичные результаты были получены и на основе анализа данных наблюдений космического аппарата TRACE (Reale, Peres, 2000). Малый градиент температуры на корональном участке магнитной петли означает, что источник нагрева должен находиться в тепловом балансе с радиационными потерями, поскольку при иных условиях невозможно обеспечить наблюдаемую квазистационарность магнитной петли на протяжении значительного временного интервала (по крайней мере, на протяжении нескольких часов). Расчеты, выполненные в Zaitsev, Shibasaki (2005), показывают, что вероятным механизмом нагрева корональных петель является омическая диссипация электрических токов. Нагрев корональной плазмы и ускорение частиц в магнитной арке связаны с включением проводимости Каулинга (Cowling, 1957), обусловленной столкновениями ионов с нейтральными атомами. Наличие в короне даже небольшого количества (порядка $10^{-5}$ от общей массы) нейтральных атомов гелия обеспечивает рост сопротивления магнитной петли на 8-9 порядков, до аномальных значений (Holman, 1985; Sprangler, 2009), необходимых для эффективного нагрева коронального вещества за счет омической диссипации крупномасштабных электрических токов.

В предыдущем исследовании (Fursyak et al., 2020) мы обнаружили крупномасштабный распределенный электрический ток $\left(I_{\text {distr }}\right)$ в шести различных по магнитным, морфологическим параметрам и уровню вспышечной продуктивности активных областях 24-го цикла солнечной активности. Мы предположили, что распределенный ток охватывает всю АО: выходит в верхние слои атмосферы в одной ее части и замыкается через хромосферу и корону на оставшейся части области. Если это действительно так, то мы должны наблюдать связь между временными вариациями величины распределенного электрического тока и процессами в солнечной короне. Поиск наличия такой зависимости и установление характера взаимосвязи между изменениями величины распределенного тока и возможными диссипативными процессами в короне на примере крупной вспышечно-продуктивной области NOAA 12192 и является основной задачей данного исследования.

\section{2 Объект исследований и данные наблюдений}

Объект исследований - AO NOAA 12192 (рис. 1a) - наибольшая по площади и одна из наиболее активных групп пятен 24-го цикла солнечной активности (Sun et al., 2015; Chen et al., 2015; Thalmann et al., 2015; McMaken, Petrie, 2017; Jain et al., 2017). Мониторинг области был начат 22 октября 2014 года в 00:00 UT. Окончание мониторинга - 25 октября 2014 года в 23:59 UT. За время наблюдения исследуемая область находилась в пределах $\pm 30^{\circ}$ по долготе относительно центрального меридиана, что позволило минимизировать ошибки в вычислении вертикальных электрических токов и распределенного электрического тока, возникающие за счет эффекта проекции.

Исходными данными для вычисления электрических токов являются Spaceweather HMI Active Region Patch (SHARP, Bobra et al., 2014; Hoeksema et al., 2014) магнитограммы компонент вектора магнитного поля исследуемой AO, доступные на интернет-ресурсе Joint Science Operations Center $(\mathrm{JSOC})^{1}$ (серия hmi.sharp_720s, временное разрешение 12 минут). Магнитограммы получены инструментом Helioseismic and Magnetic Imager (Scherrer et al., 2012), установленным на борту Solar Dynamics Observatory (Pesnell et al., 2012).

Данными для вычисления суммарной интенсивности ультрафиолетового излучения АО являются фотогелиограммы, получаемые инструментом AIA (Lemen et al., 2012) на борту SDO и доступные на вышеуказанном ресурсе JSOC. Нами использованы фотогелиограммы Солнца в четырех диапазонах волн УФ-излучения: $94 \AA, 193 \AA, 304 \AA$ (серия aia.lev1_euv_12s, данные с временным разрешением 12 с) и $1600 \AA$ (серия aia.lev1_uv_24s, данные с временным разрешением 24 c).

Для оценки уровня вспышечной активности использовались данные GOES-15 (Geostationary Operational Environmental Satellite $)^{2}$ о потоке рентгеновского излучения в диапазоне $1-8 \AA$ на орбите Земли.

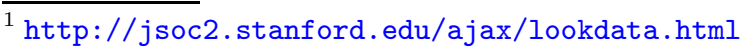

2 https://www.ngdc.noaa.gov/stp/satellite/goes/index.html
} 


\section{3 Основы методики вычисления вертикальных электрических токов, распределенного тока и интенсивности ультрафиолетового излучения в $\mathrm{AO}$}

Вычисление вертикальных электрических токов выполнено нами интегральным методом (Абраменко, Гопасюк, 1987; Fursyak, 2018) на базе магнитограмм компонент вектора поперечного магнитного поля АO:

$$
\left(I_{z}\right)_{i, j}=\frac{1}{\mu_{0}} \int_{L} \boldsymbol{B}_{t} d r,
$$

где: $\mu_{0}=4 \pi \cdot 10^{-7} \Gamma_{\text {н м }}{ }^{-1}$ - магнитная постоянная; $\boldsymbol{B}_{t} \equiv\left(B_{x}, B_{y}\right)$ - вектор поперечного магнитного поля $\mathrm{AO} ; d r$ - элемент интегрирования, равный по величине размеру пикселя на магнитограмме $\mathrm{HMI} / \mathrm{SDO}$ ( 363 км); $i, j$ - координаты пикселя на магнитограмме, для которого вычисляется электрический ток.

Интеграл в правой части выражения (1) вычислялся приближенно, с использованием метода Симпсона. Контур $L$ имел прямоугольную форму и размер $5 \times 5$ пикселей. Направление обхода контура при интегрировании осуществлялось против часовой стрелки. Таким образом, мы получали значения величины интегрального тока через контур $L$. Усреднение тока по площади контура дает нам оценку плотности вертикального тока в центральном пикселе этого контура. Вычисляя значения вертикального тока для каждого пикселя SHARP-магнитограммы, были построены карты распределения плотности вертикального тока в АО в заданный момент времени (рис. 1c).

Для обнаружения распределенного электрического тока в АО была использована косвенная методика, детально описанная в Fursyak et al. (2020), так как карты распределения плотности вертикального тока не позволяют выявить наличие крупномасштабных, распределенных по большой площади токовых структур. С физической точки зрения метод достаточно прост и суть его сводится к тому, что электрический ток создает вокруг себя вихревое магнитное поле в плоскости, перпендикулярной самому току, которое можно обнаружить. Сравнив наблюдаемое горизонтальное магнитное поле с восстановленным потенциальным полем АО и выделив компоненту, ортогональную направлению вектора потенциального поля, мы получим карту распределения непотенциальной компоненты поперечного поля $\left(\boldsymbol{B}_{\mathrm{t} \perp}\right)$ в АО. На таких картах можно четко увидеть регулярные вихревые структуры вектора $\boldsymbol{B}_{\mathrm{t} \perp}$ вблизи крупных пятен, обусловленные наличием крупномасштабных токов (рис. 1b). Если теперь очертить контур вокруг пятна так, чтобы внутри контура направление закрутки непотенциальной компоненты поперечного поля имело одно преобладающее направление (красная замкнутая кривая на рис. 1b), и, используя карту токов, рассчитать суммарный ток внутри такого контура (рис. $1, \mathrm{~d}$ ), то мы получим значение величины распределенного тока $I_{\text {distr }}$ в данный момент времени:

$$
I_{\mathrm{distr}}=\sum_{\mathrm{C}} j_{i, j} \cdot \Delta s
$$

Для вычисления суммарной интенсивности ультрафиолетового излучения АО в различных диапазонах были использованы соответствующие фотогелиограммы AIA/SDO. Эти фотогелиограммы представляют собой изображения полного диска Солнца размером $4096 \times 4096$ пикселей. Чтобы вычислить интенсивность УФ-излучения над интересующей нас областью, мы использовали предоставляемую на ресурсе JSOC процедуру обрезки изображения. Таким образом, мы получали участок, который по размеру приблизительно соответствует размерам SHARP-магнитограмм вектора магнитного поля АО. Суммарная интенсивность УФ-излучения в определенном канале рассчитывалась простым сложением интенсивностей излучения в каждом пикселе вырезанного участка:

$$
E_{\lambda}=\sum E_{i, j}
$$

Мы выполнили расчет интенсивности ультрафиолетового излучения в четырех диапазонах УФизлучения - $1600 \AA, 304 \AA, 193 \AA$ и $94 \AA$. Основные характеристики выбранных диапазонов указаны в таблице 1.

Сопоставление данных о динамике величины распределенного электрического тока с интенсивностью УФ-излучения АО в различных диапазонах является главной задачей данной работы. Но 

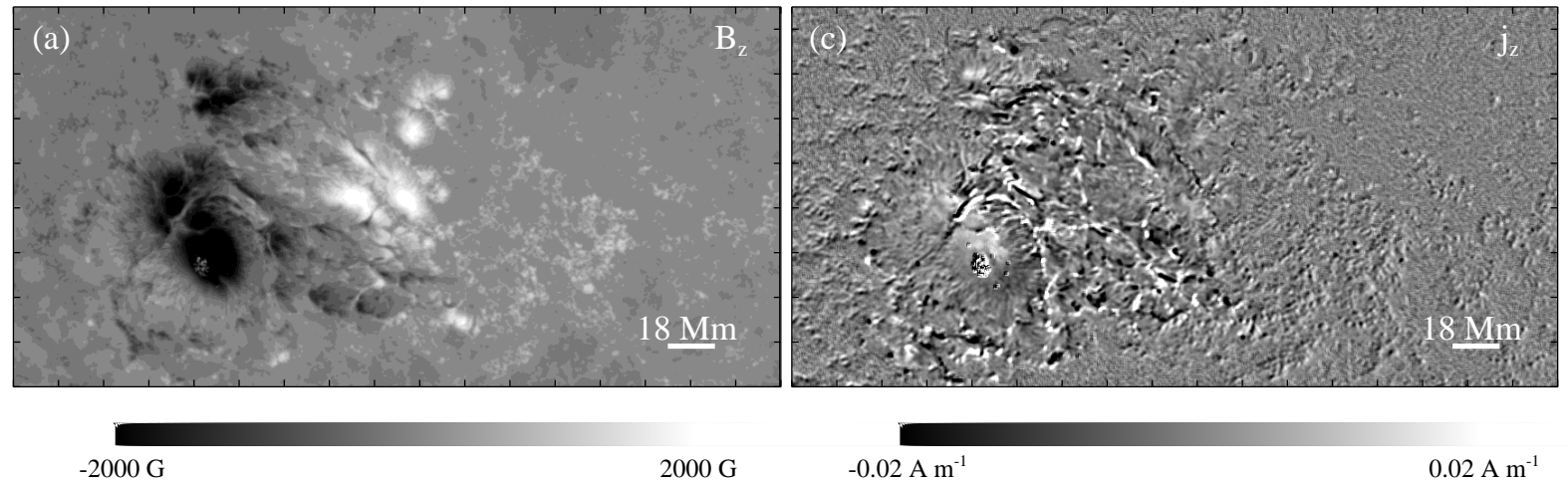

$$
-0.02 \mathrm{~A} \mathrm{~m}^{-1}
$$

$0.02 \mathrm{~A} \mathrm{~m}^{-1}$
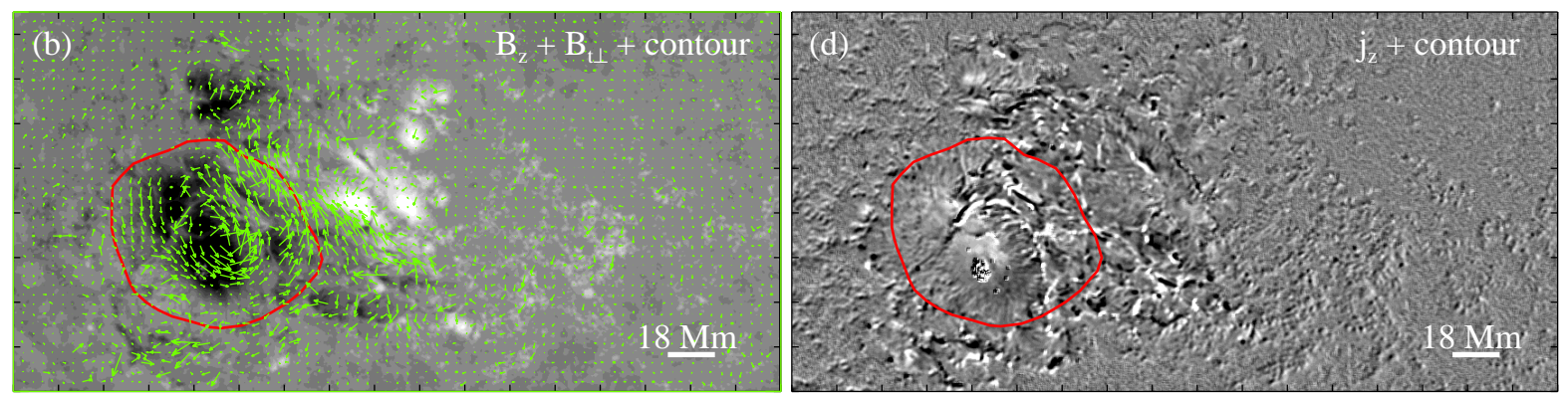

Рис. 1. (а) Магнитограмма $B_{\mathrm{z}}$-компоненты вектора магнитного поля AO NOAA 12192, полученная 22 октября 2014 года в 00:00 UT прибором HMI/SDO. Карта градуирована от -2000 Гс (черное) до 2000 Гс (белое), соответствующая шкала указана внизу изображения. (b) $B_{\mathrm{z}}$-компонента магнитного поля (фон) с нанесенными азимутами вектора непотенциальной компоненты горизонтального магнитного поля области $\boldsymbol{B}_{\mathrm{t} \perp}$ (зеленые стрелки). Длина стрелки пропорциональна величине непотенциальной компоненты поля. Красная замкнутая кривая вокруг развитого хвостового пятна S-полярности отображает контур, внутри которого преобладает одно направление закрутки вектора $\boldsymbol{B}_{\mathrm{t} \perp}$ (в данном случае - против часовой стрелки). Внутри данного контура по карте распределения плотности вертикального электрического тока (см. (d)) вычислялась величина распределенного электрического тока (более детально см. текст и Fursyak et al. (2020)). (c) Карта распределения величины плотности вертикального электрического тока в АO NOAA 12192 на момент времени 22 октября 2014 года 00:00 UT, вычисленная на основе данных о горизонтальных компонентах магнитного поля области. Карта градуирована от $-0.02 \mathrm{~A} \mathrm{~m}^{-2}$ (черное) до $0.02 \mathrm{~A} \mathrm{м}^{-2}$ (белое), соответствующая шкала указана внизу изображения. На всех изображениях север вверху, запад справа

прежде чем сравнивать данные, необходимо, чтобы они относились к одному и тому же интервалу времени и одному и тому же участку на Солнце. Основной проблемой является то, что прибор AIA/SDO выдает информацию с временным разрешением $12 / 24$ с, а HMI/SDO - с временным разрешением 720 с (12 минут). Из изначального временного массива нами были отобраны только те фотогелиограммы, которые получены в моменты времени, максимально близкие ко времени получения прибором HMI/SDO магнитограмм компонент вектора магнитного поля.

Таблица 1. Основные характеристики четырех анализируемых диапазонов УФ-излучения

\begin{tabular}{rccc}
\hline Канал AIA/SDO & Источник & Характерная температура & Область солнечной атмосферы \\
\hline $1600 \AA$ & C IV, континуум & $100000 \mathrm{~K}, 5000 \mathrm{~K}$ & Переходная область, верхняя фотосфера \\
$304 \AA$ & Не II & $50000 \mathrm{~K}$ & Хромосфера и переходная область \\
$193 \AA$ & Fe XII & $1200000 \mathrm{~K}$ & Корона \\
$94 \AA$ & Fe XVIII & $6300000 \mathrm{~K}$ & Вспышечные области в короне \\
\hline
\end{tabular}

Выбранные данные AIA/SDO были подвергнуты дальнейшей фильтрации. Цель фильтрации убрать некорректные измерения. Из массива информации были убраны данные, получаемые ежедневно в 18:00 UT (в это время происходит калибровка приборов SDO и прибор AIA выдает значения 
суммарной интенсивности излучения, близкие к нулю). Также особое внимание уделялось данным, получаемым прибором AIA/SDO во время вспышек. Поскольку во время вспышечных процессов наблюдается интенсивное излучение в ультрафиолете, динамического диапазона в некоторых каналах прибора AIA/SDO оказывается недостаточно для адекватного отражения уровня излучения и прибор начинает работать в другой моде. Искаженные данные мы также не учитывали. Таким образом, для построения графиков временных зависимостей, а также корреляционных графиков, были использованы только те данные AIA/SDO, в которых отсутствуют какие-либо искажения, связанные с артефактами самого прибора, и в то же время совпадающие по времени с данными, получаемыми $\mathrm{HMI} / \mathrm{SDO}$.

В нашем случае из четырех выбранных для анализа диапазонов УФ-излучения наибольшее количество искажений наблюдалось в канале $193 \AA$ А. Данные, получаемые в других диапазонах, были приведены к единой системе по диапазону $193 \AA$ А. По этой причине наблюдается существенная скважность информации об интенсивности УФ-излучения во всех четырех каналах за время наблюдения исследуемой $\mathrm{AO}$ (см. рис. 3).

\section{4 Результаты}

В предыдущем исследовании (Fursyak et al., 2020) нами было показано существование в АО крупномасштабных токовых структур. Низкий (значительно меньше $1 \%$ ) разбаланс вертикальных токов, рассчитанный по всей площади АО, позволил сделать предположение о том, что распределенный электрический ток уходит в верхние слои атмосферы Солнца в одной части АО, замыкаясь через хромосферу и корону на оставшейся ее части. Если это так, то вычисленный нами распределенный ток, вынося в верхние слои атмосферы Солнца большое количество свободной магнитной энергии, должен быть связан со вспышками и иными диссипативными процессами в короне. В той же работе на примере 6 АО была проанализирована связь между временными вариациями величины распределенного электрического тока и уровнем вспышечной активности. Показано, что абсолютные значения величины распределенного тока и амплитуда его изменений больше в областях с более высоким уровнем вспышечной продуктивности.

Здесь мы рассматриваем распределенный электрический ток и его динамику в АO NOAA 12192. Как следует из рис. 1a, с, наиболее развитая вихревая структура непотенциальной компоненты вектора горизонтального магнитного поля, обусловленная наличием крупномасштабного электрического тока, была обнаружена вокруг крупного пятна S-полярности в хвостовой части АО. По направлению закрутки вектора $\boldsymbol{B}_{\mathrm{t} \perp}$ (против часовой стрелки) мы установили, что знак распределенного тока должен быть положительным (ток направлен вверх, на наблюдателя), а, следовательно, данная токовая структура связана не с развитыми пятнами N-полярности, находящимися к западу от рассматриваемого пятна (в которых закрутка вектора $\boldsymbol{B}_{\mathrm{t} \perp}$ также предполагает наличие положительного тока), а с обширной областью слабых магнитных полей N-полярности на юго-западе. Наше предположение хорошо согласуется с данными теоретических расчетов. Так, в работе Jiang et al. (2016) показано наличие крупномасштабной токовой структуры, уходящей в корону на высоту порядка 46 Мм и связанную, с одной стороны, с крупным пятном S-полярности, а с другой - с областью слабых магнитных полей N-полярности, находящихся к юго-западу от большого пятна.

Мы проанализировали временные изменения вычисленного нами распределенного электрического тока и сопоставили его временные вариации со вспышками, зафиксированными в исследуемой области за время мониторинга (рис. 2). Из графика видно, что наиболее мощные вспышки, наблюдавшиеся в АО на протяжении суток 22 октября 2014 года, а также 24-25 октября 2014 года, можно связать с локальными максимумами значений величины $I_{\mathrm{distr}}$. Во временной промежуток 23 октября - первой половине 24 октября величина распределенного тока в области достигла минимальных значений за все время мониторинга (порядка 40·10 12 А). В этот же временной интервал можно отметить и отсутствие вспышечных процессов рентгеновского класса выше М5.0.

Большие значения величины распределенного электрического тока указывают на большой запас выносимой в верхние слои солнечной атмосферы свободной магнитной энергии. Так, в работе Абраменко, Гопасюк (1987) была выполнена приблизительная оценка энергии, выносимой в корону глобальным (крупномасштабным) электрическим током величиной порядка $10^{12}$ А. Получены значения порядка $10^{32}$ эрг, что сопоставимо с полной энергией, выделяемой во время мощной солнечной вспышки. В нашем последнем исследовании (Fursyak et al., 2020) даже в АО с низкой вспышечной 
активностью выявлено наличие распределенных токов величиной $\pm(10-20) \cdot 10^{12} \mathrm{~A}$, что (по грубым оценкам) соответствует энергии $10^{32}-10^{33}$ эрг. Минимальные значения величины распределенного тока в области NOAA 12192 за время мониторинга AO, как мы видим из рис. 2, близки к значениям $40 \cdot 10^{12} \mathrm{~A}$, что уже эквивалентно энергии в $10^{33}-10^{34}$ эрг. Несмотря на такой колоссальный запас магнитной энергии в короне, в этот временной интервал мощных вспышек в исследуемой АО не наблюдается. Это означает, что большая часть энергии, носителями которой являются электрические токи в короне, реализуется не в виде вспышек, а посредством иных диссипативных процессов, а во вспышках выделяется лишь небольшое ее количество. Данное предположение подтверждается более ранними исследованиями других авторов (Zaitsev et al., 1998; Zaitsev, Stepanov, 2008), которые утверждают, что во время солнечной вспышки выделяется не более $5-10 \%$ энергии, запасенной в электрическом токе. Одним из наиболее вероятных механизмов диссипации энергии токов в короне, не связанных со вспышечным энерговыделением, может быть нагрев коронального вещества электрическими токами, протекающими в верхних слоях солнечной атмосферы.

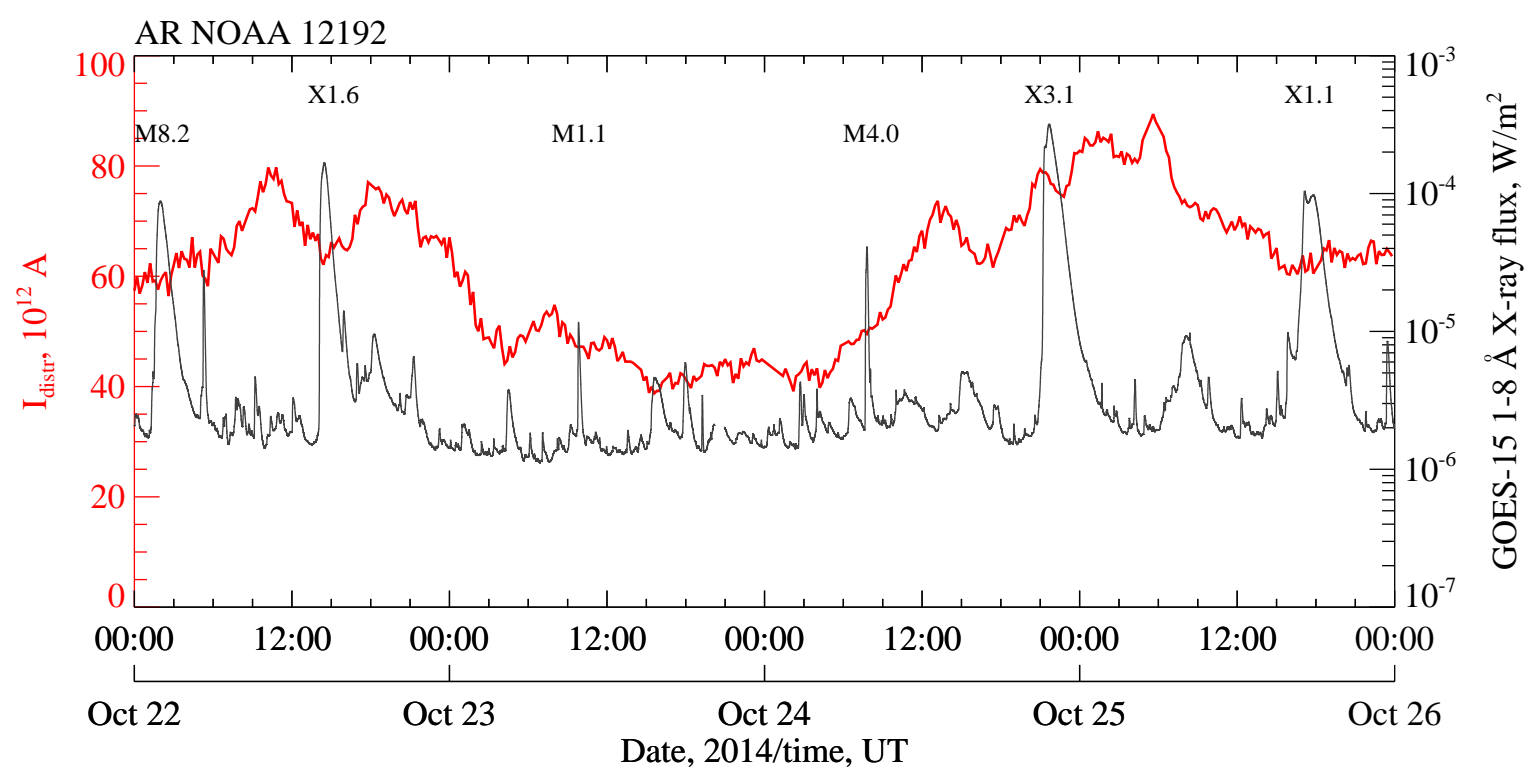

Рис. 2. Динамика величины распределенного электрического тока в AO NOAA 12192 за время мониторинга (красная кривая). Серой кривой обозначены временные вариации потока рентгеновского излучения в диапазоне длин волн 1-8 А на орбите Земли (данные космического аппарата GOES-15). Указаны рентгеновские классы наиболее мощных вспышек, ассоциированных с исследуемой АО

Исследование связи временных изменений величины распределенного тока и уровня вспышечной активности, представленных на рис. 2, позволяет сделать еще два важных вывода. В частности, можно отметить более плавный характер временных вариаций величины распределенного тока по сравнению с потоком рентгеновского излучения. Объяснить это можно тем, что распределенный ток является крупномасштабной структурой, существующей на протяжении значительного временного интервала и охватывающей как всю АО, так и все слои атмосферы Солнца. В то же время вспышка является явлением менее масштабным как в пространственном, так и во временном интервале.

Также из рис. 2 следует отсутствие резких изменений величины распределенного тока во время вспышек. В то же время многие модели предполагают непосредственное участие электрических токов и токовых слоев во вспышечном процессе (Alfven, Carlqvist, 1967; Parker, 1973; Sokolov, Kosovichev, 1978; Heyvaerts et al., 1977; Priest, Forbes, 2002; Sui, Holman, 2003; Li et al., 2018). Это несоответствие может быть объяснено высокой индуктивностью токопроводящей петли (Alfven, Carlqvist, 1967; Khodachenko et al., 2003) и, следовательно, медленным откликом системы крупномасштабных электрических токов на события в верхних слоях солнечной атмосферы. 

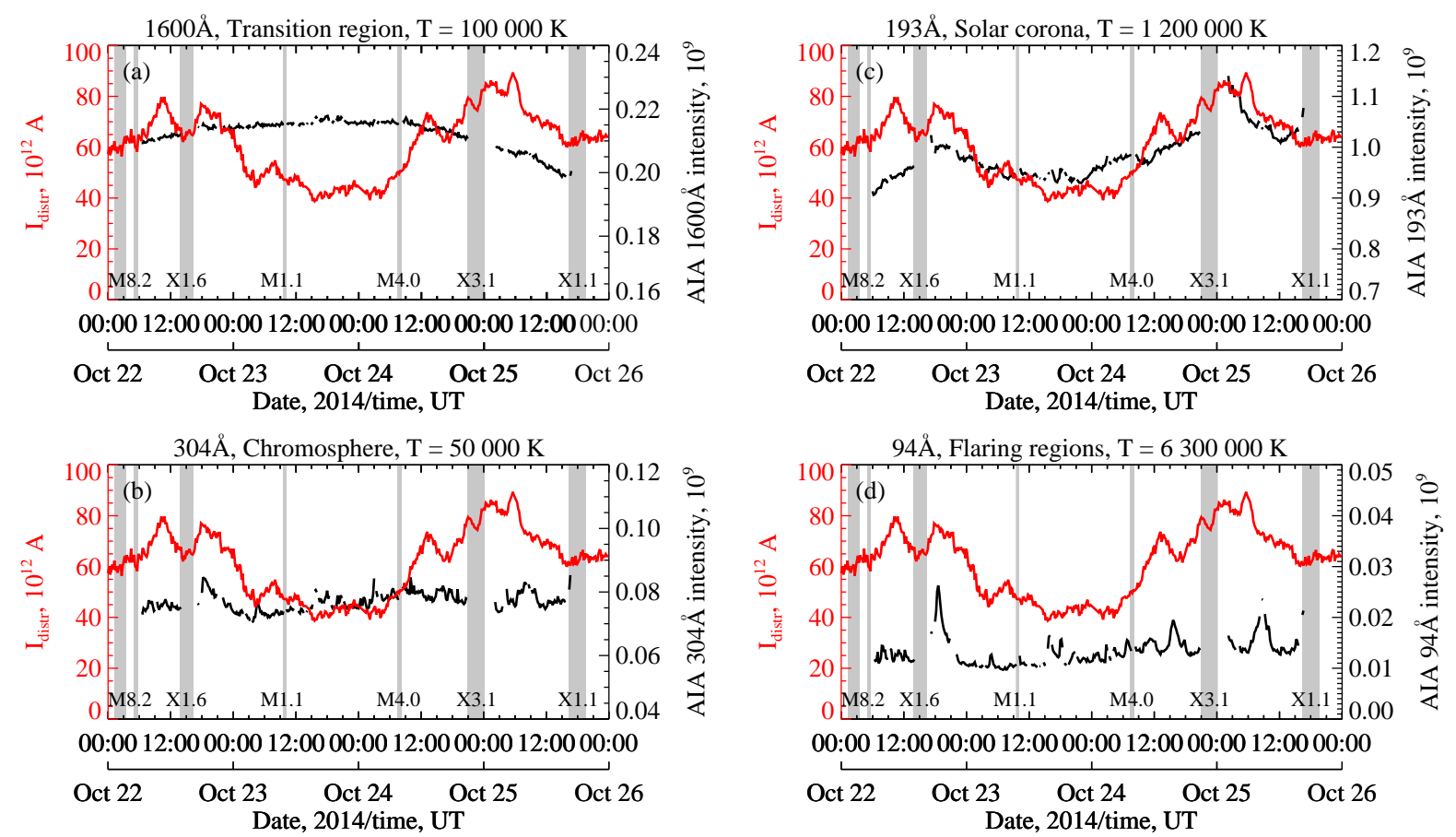

Рис. 3. Временные изменения величины распределенного электрического тока (красная кривая) и суммарной интенсивности ультрафиолетового излучения АО (черная кривая) в каналах $1600 \AA$ (a), $304 \AA$ (b), $193 \AA$ (c), $94 \AA$ (d). Данные об интенсивности УФ-излучения получены на основе данных прибора AIA/SDO. Большая скважность полученной информации обусловлена особенностями работы прибора (более детально см. текст). Серыми вертикальными полосами обозначены временные интервалы, на протяжении которых поток рентгеновского излучения от исследуемой АO в диапазоне 1-8 А (по данным GOES-15) превышал значение $10^{-5} \mathrm{Bт} \mathrm{м}^{-2}$ (эквивалент вспышки рентгеновского класса M1.0). Внизу графиков указаны наиболее мощные вспышечные процессы, ассоциированные с исследуемой областью

На рис. 3 представлены кривые изменения величины $I_{\text {distr }}$ и интенсивности УФ-излучения в четыpex каналах инструмента AIA/SDO. Также были получены корреляционные кривые, отображающие характер взаимосвязи величин $I_{\text {distr }}$ и интенсивности УФ-излучения в каждом из рассматриваемых диапазонов (рис. 4).

Чтобы убедиться в правильности нашего предположения о том, что обнаруженный распределенный электрический ток уходит в верхние слои солнечной атмосферы и может участвовать в нагреве коронального вещества, мы сопоставили временные изменения величины $I_{\mathrm{distr}}$ и интенсивности УФ-излучения (рис. 3) в четырех диапазонах длин волн (см. таблицу 1), стратифицированных по температуре и, соответственно, по высоте. Также были получены корреляционные графики, отображающие характер взаимосвязи величин $I_{\operatorname{distr}}$ и интенсивности УФ-излучения в каждом из рассматриваемых диапазонов (рис. 4).

Из графиков, представленных на рис. 3, видно, что для каналов $1600 \AA$ и $304 \AA$ временные вариации величины распределенного тока не связаны с изменениями интенсивности УФ-излучения. Так, изменение интенсивности УФ-излучения в канале $1600 \AA$ можно объяснить эффектом потемнения к краю диска. Слабую взаимосвязь между величиной распределенного тока и интенсивностью УФизлучения в канале $304 \AA$ подтверждают и корреляционные графики: для диапазона $304 \AA$ (рис. 4b) коэффициент корреляции между анализируемыми величинами составляет 0.22.

Несмотря на отсутствие явной связи между величиной распределенного тока и интенсивностью УФ-излучения в канале $94 \AA$ (как следует из рис. $4 \mathrm{~d}, k=0.45$ ), на графике временных вариаций исследуемых величин (рис. 3d) можно наблюдать незначительные по амплитуде хаотичные изменения интенсивности излучения на протяжении всего времени мониторинга АО, не связанные явно со вспышечными процессами в исследуемой области. При этом наиболее значимые пики интенсивности 

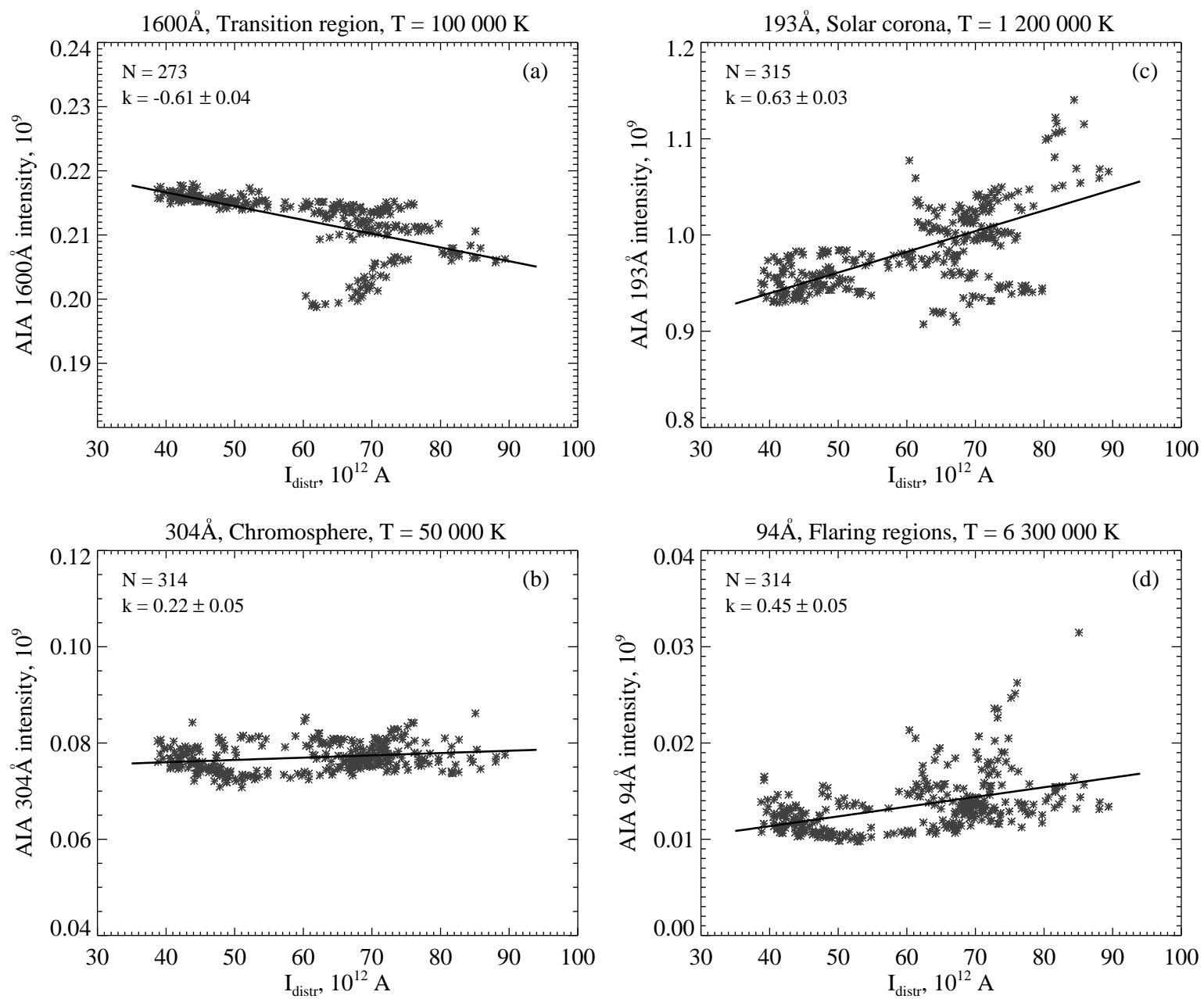

Рис. 4. Корреляционные графики между величиной распределенного электрического тока (горизонтальная шкала) и суммарной интенсивностью УФ-излучения АО (вертикальная шкала) в каналах $1600 \AA$ (а), $304 \AA$ (b), $193 \AA$ (c), $94 \AA$ (d). Коэффициенты корреляции в каждом случае указаны в левом верхнем углу соответствующих графиков

УФ-излучения близки по времени к локальным максимумам величины распределенного тока, отставая от них по временной шкале на 1-4 часа. Это наблюдение может являться признаком не только участия электрических токов в диссипативных процессах в верхних слоях солнечной атмосферы, но и правильности высказанного предположения о высокой индуктивности магнитной токонесущей трубки.

Иная картина наблюдается для канала $193 \AA$ (рис. 3c). Несмотря на большую скважность данных, можно отметить два максимума интенсивности УФ-излучения в данном диапазоне. Один из них наблюдался на протяжении суток 22 октября 2014 года, второй - 24-25 октября 2014 года. Максимумы значений интенсивности УФ-излучения в канале $193 \AA$ совпадают по времени с максимумами величины $I_{\text {distr. }}$ График на рис. 4c показывает наличие хорошей корреляционной зависимости между анализируемыми величинами (коэффициент корреляции $k=0.63$ ).

Анализ временных вариаций величины распределенного электрического тока и интенсивности УФ-излучения в четырех разных диапазонах (рис. 3), а также корреляционные зависимости между этими величинами позволяют предположить, что глобальный ток может играть существенную роль в нагреве корональных петель. K сожалению, нет однозначной простой связи между температурой плазмы в короне и интенсивностью УФ-излучения в определенных спектральных линиях. В 
общем случае проблема измерения температуры в короне требует решения задачи инверсии дифференциальной меры эмиссии ряда спектральных линий (например, Morgan and Pickering, 2019). Tем не менее, наблюдающаяся хорошая корреляция (рис. 4c) между величиной распределенного тока и интенсивностью УФ-излучения в канале $193 \AA$ (рис. 3c) может свидетельствовать о причастности омической диссипации токов к энерговыделению в ультрафиолете. Если предположение верно, этот результат хорошо согласуется с концепцией эквивалентного электрического (LRC) контура токонесущей петли, рассматриваемой в работах (Zaitsev, Khodachenko, 1997; Zaitsev et al., 1998; Zaitsev, Stepanov, 2008; Stepanov et al., 2012; Zaitsev, Kronshtadtov, 2016). Согласно данной модели, крупномасштабные электрические токи должны существовать в короне и участвовать в нагреве коронального вещества.

Отсутствие явной связи между временными вариациями интенсивности УФ-излучения в других каналах и величиной $I_{\text {distr }}$ (рис. $4 \mathrm{a}, \mathrm{b}, \mathrm{d}$ ) может указывать на то, что нагрев корональной плазмы за счет омической диссипации электрических токов эффективен только в определенном температурном диапазоне. Связано это с проводимостью плазмы, которая сильно зависит от температуры $\left(\sigma T^{3 / 2}\right)$. При низких (характерных для хромосферы и переходного слоя) и высоких (горячая вспышечная плазма, высокотемпературная плазма в короне) температурах эффективность нагрева корональных структур за счет омической диссипации токов существенно снижается.

Отметим также, что, поскольку энергия, запасенная в электрическом токе, пропорциональна его квадрату $\left(W_{\mathrm{m}} \sim I^{2}\right)$, мы рассмотрели и корреляционную зависимость между интенсивностями УФизлучения в диапазонах $1600 \AA, 304 \AA, 193 \AA, 94 \AA$ и квадратом величины $I_{\mathrm{distr}}$. Однако данные изменения не привели к ожидаемым результатам: корреляция изменилась лишь на несколько процентов.

$\mathrm{K}$ примеру, зависимость $I_{\mathrm{distr}}^{2}-E_{193 \AA}$ показала корреляцию $k=0.65$, в то время как коэффициент корреляции для зависимости $I_{\mathrm{distr}}-E_{193 \AA}$ составляет 0.63 (рис. 4c). Подобный результат позволяет предположить, что, по-видимому, вспышки и омическая диссипация являются не единственными процессами, в которые вовлечены крупномасштабные электрические токи в короне.

\section{5 Выводы и обсуждение}

Обнаружение крупномасштабного распределенного электрического тока и сопоставление его динамики с изменением уровня вспышечной продуктивности области и временными вариациями УФизлучения в разных диапазонах длин волн на примере АО NOAA 12192 позволяют сделать следующие выводы:

1. Наличие хорошей корреляции $(k=0.63)$ между величиной распределенного электрического тока $I_{\text {distr }}$ и интенсивностью УФ-излучения в канале AIA/SDO $193 \AA$ является возможным указанием на то, что обнаруженный нами крупномасштабный электрический ток действительно уходит в корону. Помимо этого, омическая диссипация крупномасштабных токов, наряду с другими процессами, может являться одним из важных механизмов нагрева корональных петель.

2. Грубая оценка показывает, что распределенный электрический ток выносит в верхние слои солнечной атмосферы энергию порядка $10^{33}-10^{34}$ эрг, что значительно превышает суммарную энергию даже мощных вспышек. По-видимому, большая часть энергии крупномасштабных электрических токов реализуется в виде иных диссипативных процессов в короне.

3. Отсутствие резких изменений величины распределенного тока во время мощных вспышечных процессов, а также наличие небольших по амплитуде пиков интенсивности УФ-излучения в канале $94 \AA$, связанных с локальными максимумами величины распределенного тока, имеет место благодаря высокой индуктивности магнитной токонесущей трубки.

4. Отсутствие корреляции между динамикой распределенного тока и УФ-излучением в каналах $1600 \AA, 304 \AA$ и слабую корреляцию в канале $94 \AA$ можно объяснить сильной зависимостью проводимости плазмы от температуры. Определенный вклад в нагрев корональных структур омическая диссипация электрических токов может вносить только при температурах, характерных для короны (порядка 0.5-2.5 MK). При более низких или более высоких температурах (например, в горячей вспышечной плазме) этот механизм нагрева теряет свою эффективность и нагрев коронального вещества происходит за счет других процессов.

Отсутствие значительного улучшения корреляционной зависимости между квадратом величины распределенного тока и интенсивностями излучения в различных диапазонах длин волн УФ- 
излучения по сравнению с результатами, представленными на рис. 3 и рис. 4, позволяет предположить, что, по-видимому, вспышки и омическая диссипация являются не единственными процессами, в которых задействованы крупномасштабные электрические токи в верхних слоях солнечной атмосферы.

Благодарности. Исследование распределенных электрических токов и их связи со вспышечной продуктивностью АО выполнено при поддержке гранта Российского научного фонда (РНФ) № 1812-00131; анализ временных вариаций интенсивности УФ-излучения в короне над исследуемой АО осуществлен в рамках научно-исследовательской работы (НИР) ОФССС КрАО РАН № 0831-20190006 "Общие и локальные характеристики Солнца". Авторы признательны рецензенту за интерес к статье и полезные замечания, которые в значительной степени помогли улучшить данную работу.

\section{Литература}

Абраменко В.И., Гопасюк С.И., 1987. Изв. Крымск. Астрофиз. Обсерв. Т. 76. С. $147-168$. [Abramenko V.I., Gopasyuk S.I., 1987. Izv. Krymsk. Astrofiz. Observ., vol. 76, pp. 147-168. (In Russ.)] Абраменко В.И., Гопасюк С.И., Огирь М.Б., 1991. Изв. Крымск. Астрофиз. Обсерв. Т. 83. С. 3-11. [Abramenko V.I., Gopasyuk S.I., Ogir' M.B., 1991. Izv. Krymsk. Astrofiz. Observ., vol. 83, pp. 3-11. (In Russ.)]

Alfven H., Carlqvist P., 1967. Solar Phys., vol. 1, pp. 220-228.

Antonucci E., Alexander D., Culhane J.L., et al., 1998. In Strong K.T., et al. (Eds), The Many Faces of the Sun: A Summary of the Results from NASA's Solar Maximum Mission. Berlin: Springer, p. 331.

Aschwanden M.J., Alexander D., Hurlburt N., et al., 2000. Astrophys. J., vol. 531, pp. 1129-1149.

Aschwanden M.J., Newmark J.S., Delabourdiniere J.-P., et al., 1999. Astrophys. J., vol. 515, pp. $842-867$. Aulanier G., Demoulin P., Grappin R., 2005. Astron. Astrophys., vol. 430, pp. 1067-1087.

Bobra M.G., Sun X., Hoeksema J.T., et al., 2014. Solar Phys., vol. 289, pp. 3549-3578.

Bornmann P.L., 1998. In Strong K.T., et al. (Eds), The Many Faces of the Sun: A Summary of the Results from NASA's Solar Maximum Mission. Berlin: Springer, p. 301.

Chen H., Zhang J., Ma S., et al., 2015. Astrohys. J., vol. 808, p. L24.

Cheung M.C.M., Isobe H., 2014. Living Rev. Solar Phys., vol. 11, article ID 3.

Cowling T.G., 1957. Magnetohydrodynamics. London: Interscience Publ., Great Britain.

Dalmasse K., Aulanier G., Demoulin P., Kliem B., Török T., Pariat E., 2015. Astrophys. J., vol. 810, article ID 17.

Fursyak Yu.A., 2018. Geomagnetism and Aeronomy, vol. 58, pp. 1129-1135.

Fursyak Yu.A., Kutsenko A.S., Abramenko V.I., 2020. Solar Phys., vol. 295, article ID 19.

Galeev A.A., Rosner R., Serio S., Vaiana G.S., 1981. Astrophys. J., vol. 243, pp. 301-308.

Georgoulis M.K., Titov V.S., Mikic Z., 2012. Astrophys. J., vol. 761, article ID 61.

Gosain S., Demoulin P., Lopez Fuentes M., 2014. Astrophys. J., vol. 793, article ID 15.

Heyvaerts J., Priest E.R., Rust D.M., 1977. Astrophys. J., vol. 216, pp. 123-137.

Hoeksema J.T., Liu Y., Hayashi K., et al. 2014. Solar Phys., vol. 289, pp. 3483-3530.

Hollweg J.V., 1984. Astrophys. J., vol. 277, pp. 392-403.

Holman G.D., 1985. Astrophys. J., vol. 293, pp. 584-594.

Ionson J., 1984. Astrophys. J., vol. 276, pp. 357-368.

Jain K., Tripathy S.C., Hill F., 2017. Astrophys. J., vol. 849, article ID 94.

Jiang, C., Wu, S.T., Yurchyshyn, V., 2016. Astrophys. J., vol. 828, article ID 62.

Khodachenko M., Haerendel G., Rucker H.O., 2003. Astron. Asrophys., vol. 401, pp. 721-732.

Khodachenko M.L., Zaitsev V.V., 2002. Astrophys. Space. Sci., vol. 279, pp. 389-410.

Kontogiannis I., Georgoulis M.K., Park S.-H., Guerra J.A., 2017. Solar Phys., vol. 292, article ID 159.

Leka K.D., Canfield R.C., McClymont A.N., van Driel-Gesztelyi L., 1996. Astrophys. J., vol. 462, pp. 547560 .

Lemen J.R., Title A.M.,Akin D.J., et al., 2012. Solar Phys., vol. 275, pp. 17-40.

Li Y., Xue J.C., Ding M.D., et al., 2018. Astrophys. J. Lett., vol. 853, p. L15.

Liu Y., Sun X., Török T., Titov V.S., Leake J.E., 2017. Astrophys. J., vol. 846, p. L6.

Longcope D.W., Welsch B.T., 2000. Astrophys. J., vol. 545, pp. 1089-1100. 
McClymont A.N., Fisher G.H., 1989. Washington DC American Geophysical Union Geophysical Monograph Series, vol. 54, pp. 219-225.

McMaken T.C., Petrie G.J.D., 2017. Astrophys. J., vol. 840, article ID 100.

Melrose D.B., 1991. Astrophys. J., vol. 381, pp. 306-312.

Melrose D.B., 1995. Astrophys. J., vol. 451, pp. 391-401.

Melrose D.B., Dulk G.A., 1984. Astrophys. J., vol. 282, pp. 308-315.

Morgan H., Pickering J., 2019. Solar Phys., vol. 294, article ID 135.

Parker E.N., 1973. Astrophys. J., vol. 180, pp. 247-252.

Parker E.N., 1988. Astrophys. J., vol. 330, pp. 474-479.

Parker E.N., 1996. Astrophys. J., vol. 471, pp. 485-496.

Priest E.R., Forbes T.G., 2002. Astron. Astrophys. Rev., vol. 10, pp. 313-377.

Pesnell W.D., Thompson B.J., Chamberlin P.C., 2012. Solar Phys., vol. 275, pp. 3-15.

Reale F., Peres G., 2000. Astrophys. J., vol. 528, p. L45.

Scherrer P.H., Schou J., Bush R.I., et al., 2012. Sol. Phys., vol. 275, pp. 207-227.

Severny A.B., 1964. Space Sci. Rev., vol. 3, pp. 451-486.

Sokolov V.S., Kosovichev A.G., 1978. Solar Phys., vol. 57, pp. 73-79.

Spicer D.C., 1991. In Ulmschneider P., et al. (Eds), Mechanisms of Chromospheric and Coronal Heating. Berlin: Springer-Verlag, p. 547.

Sprangler S.R., 2009. Nonlin. Processes Geophys., vol. 16, pp. 443-452.

Stenflo J.O., 1969., Solar Phys., vol. 8, pp. 115-118.

Stepanov A.V., Zaitsev V.V., Nakariakov V.M., 2012. Coronal Seismology. Wienheim: WILEY-VCH, Germany.

Sui L., Holman G.D., 2003. Astrophys. J., vol. 596, p. L251.

Sun X., Bobra M.G., Hoeksema J.T., et al., 2015. Astrophys. J. Lett., vol. 804, p. L28.

Thalmann J.K., Su Y., Temmer M., Veronig A.M., 2015. Astrophys. J. Lett., vol. 801, p. L23.

Török T., Kliem B., 2003. Astron. Astrophys., vol. 406, pp. 1043-1059.

Török T., Leake J.E., Titov V.S., et al., 2014. Astrophys. J., vol. 782, p. L10.

Zaitsev V.V., Khodachenko M.L., 1997. Radiophys. Quantum Electron., vol. 40, pp. 114-138.

Zaitsev V.V., Kronshtadtov P.V., 2016. Radiophys. Quantum Electron., vol. 59, pp. 169-176.

Zaitsev V.V., Shibasaki K., 2005. Astronomy Reports, vol. 49, pp. 1009-1017.

Zaitsev V.V., Stepanov A.V., 2008. Uspekhi Fiz. Nauk, vol. 51, pp. 1123-1160.

Zaitsev V.V., Stepanov A.V., Urpo S., Pohjolainen S., 1998. Astron. Astrophys., vol. 337, pp. 887-896. 


\title{
Distributed electric current and its relation to ultraviolet radiation of the active region
}

\author{
Yu.A. Fursyak, A.S. Kutsenko \\ Crimean Astrophysical Observatory, Nauchny, 298409 \\ yuriy_fursyak@mail.ru
}

\begin{abstract}
We utilized vector magnetic field magnetograms acquired by the Helioseismic and Magnetic Imager (HMI) onboard the Solar Dynamics Observatory (SDO) to calculate vertical electric currents in the active region (AR) NOAA 12192. The AR was tracked between 2014 October 22 and 2014 October 25 with $720 \mathrm{~s}$ cadence. We revealed the presence of a large-scale electric current structure - a distributed electric current - with the absolute magnitude varying in the range of (40-90)·10 ${ }^{12} \mathrm{~A}$. The distributed electric current is supposed to exist throughout the entire AR, and, penetrating the upper layers of the solar atmosphere in one part of the active region, it closes through the chromosphere and corona in the remaining part. To test this assumption, we have compared the temporal variation of the magnitude of the distributed electric current with the flare activity level (using GOES-15 data), as well as with the intensity of ultraviolet radiation (UV) in the AR (using the Atmospheric Imaging Assembly (AIA/SDO) data in channels $94 \AA, 193 \AA, 304 \AA$, and $1600 \AA)$. We found that: i) Time intervals of enhanced flare activity are co-temporal with intervals of increased magnitudes of the distributed electric current. The absence of rapid changes in the magnitude of the distributed electric current during solar flares can be explained by high inductance of current-carrying magnetic loops. ii) Rough estimates of the magnetic energy carried by the distributed electric current into the corona yield the values of about $10^{33}-10^{34} \mathrm{erg}$ for AR NOAA 12192. Only a small amount of this energy is released during flare processes in the AR. Most of this energy seems to be consumed during other dissipative processes in the corona. iii) Comparison of the temporal variations of intensity in the $193 \AA$ UV-radiation channel with dynamics of the distributed electric current in the AR reveals a good positive correlation between these values (Pearson's $\mathrm{R}=0.63$ ). The absence of correlation between the distributed electric current magnitude and the intensity of UV radiation in channels $1600 \AA, 304 \AA$ and $94 \AA$ might be explained by a low efficiency of the coronal loop heating by ohmic dissipation of electric currents in the corona due to the strong dependence of plasma conductivity on the temperature. iv) Our results may support the concept of equivalent LRC contour of a current-carrying coronal magnetic loop proposed by Alfven and Carlqvist in 1967 and developed by V.V. Zaitsev, A.V. Stepanov, and others. According to this model, the large-scale electric currents must exist in the upper layers of the solar atmosphere and take part in the heating of the coronal plasma.
\end{abstract}

Key words: Sun, active regions, solar activity, electric currents, coronal heating 